\title{
Assignment of Pterin Deaminase Activity to an Enzyme of Unknown Function Guided by Homology Modeling and Docking
}

\author{
Hao Fan, ${ }_{\dagger}^{\dagger, \S, \nabla}$ Daniel S. Hitchcock, ${ }^{\|, \nabla}$ Ronald D. Seidel II, ${ }^{\#}$ Brandan Hillerich, ${ }^{\#}$ Henry Lin,
}

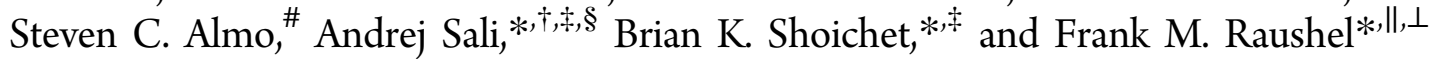

${ }^{\dagger}$ Department of Bioengineering and Therapeutic Sciences, ${ }^{\ddagger}$ Department of Pharmaceutical Chemistry, and ${ }^{\S}$ California Institute for Quantitative Biosciences, University of California, San Francisco, California 94143, United States

${ }^{\|}$Department of Biochemistry \& Biophysics, and ${ }^{\perp}$ Department of Chemistry, Texas A\&M University, College Station, Texas 77843 , United States

\#Albert Einstein College of Medicine, 1300 Morris Park Avenue, Bronx, New York 10461, United States

Supporting Information

ABSTRACT: Of the over 22 million protein sequences in the nonredundant TrEMBL database, fewer than $1 \%$ have experimentally confirmed functions. Structure-based methods have been used to predict enzyme activities from experimentally determined structures; however, for the vast majority of proteins, no such structures are available. Here, homology models of a functionally uncharacterized amidohydrolase from Agrobacterium radiobacter K84 (Arad3529) were computed on the basis of a remote template structure. The protein backbone of two loops near the active site was remodeled, resulting in four distinct active site conformations. Substrates of Arad3529 were predicted by docking of 57672 highenergy intermediate (HEI) forms of 6440 metabolites against these four homology models. On the basis of docking ranks and geometries, a set of modified pterins were suggested as candidate substrates for Arad3529. The predictions were tested by enzymology experiments, and Arad3529 deaminated many pterin metabolites

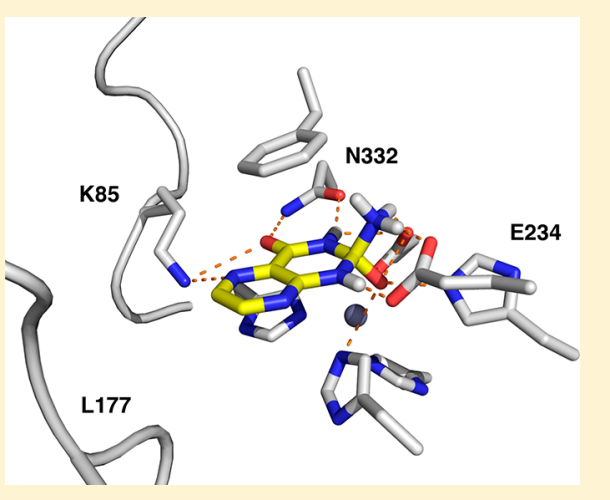
(substrate, $k_{\mathrm{cat}} / K_{\mathrm{m}}\left[\mathrm{M}^{-1} \mathrm{~s}^{-1}\right]$ ): formylpterin, $5.2 \times 10^{6}$; pterin-6-carboxylate, $4.0 \times 10^{6}$; pterin-7-carboxylate, $3.7 \times 10^{6}$; pterin, 3.3 $\times 10^{6}$; hydroxymethylpterin, $1.2 \times 10^{6}$; biopterin, $1.0 \times 10^{6}$; D- $(+)$-neopterin, $3.1 \times 10^{5}$; isoxanthopterin, $2.8 \times 10^{5}$; sepiapterin, $1.3 \times 10^{5}$; folate, $1.3 \times 10^{5}$, xanthopterin, $1.17 \times 10^{5}$; and 7,8-dihydrohydroxymethylpterin, $3.3 \times 10^{4}$. While pterin is a ubiquitous oxidative product of folate degradation, genomic analysis suggests that the first step of an undescribed pterin degradation pathway is catalyzed by Arad3529. Homology model-based virtual screening, especially with modeling of protein backbone flexibility, may be broadly useful for enzyme function annotation and discovering new pathways and drug targets.

\section{INTRODUCTION}

With increasing availability of genomic sequences, a pressing challenge in biology is a reliable assignment of function to the proteins encoded by these genomes. Functional annotation of an uncharacterized protein can be conveniently accomplished by matching its sequence to that of a characterized protein. ${ }^{1,2}$ However, this strategy is often inaccurate and imprecise. ${ }^{3,4}$ Conservatively, over $50 \%$ of the sequences in the public databases have uncertain, unknown, or incorrectly annotated functions. ${ }^{5}$ Annotation of enzymes in functionally diverse superfamilies is particularly challenging. ${ }^{6}$

A promising method to functional assignment is the identification of the substrate by docking potential substrates against the binding site of an experimentally determined enzyme structure. ${ }^{7,8}$ This method was used to predict the substrates of Tm0936 from Thermotoga maritima. ${ }^{9}$ High-energy intermediate forms of thousands of candidate metabolites were docked to the X-ray structure of Tm0936, and those highly ranked by docking energy score were tested experimentally, confirming a significant deaminase activity against $S$-adeno- sylhomocysteine (SAH). This approach has been subsequently applied to three other enzymes for activity determination. ${ }^{10-12}$ These studies suggest that structure-based docking might be a useful tool for enzyme function annotation, when an experimentally determined atomic structure of the enzyme is available.

Often, however, an enzyme of unknown function has no experimentally determined three-dimensional structure. In such situations, a three-dimensional model of the target sequence can be computed by homology modeling if a template structure of a related protein is known. ${ }^{13}$ Currently, the total fraction of protein sequences in a typical genome for which reliable homology models can be obtained varies from $20 \%$ to $75 \%$, increasing the number of structurally characterized protein sequences by more than 2 orders of magnitude relative to the PDB. ${ }^{14}$ Therefore, homology models can, in principle, greatly extend the applicability of virtual screening for ligand

Received: October 1, 2012

Published: December 20, 2012 


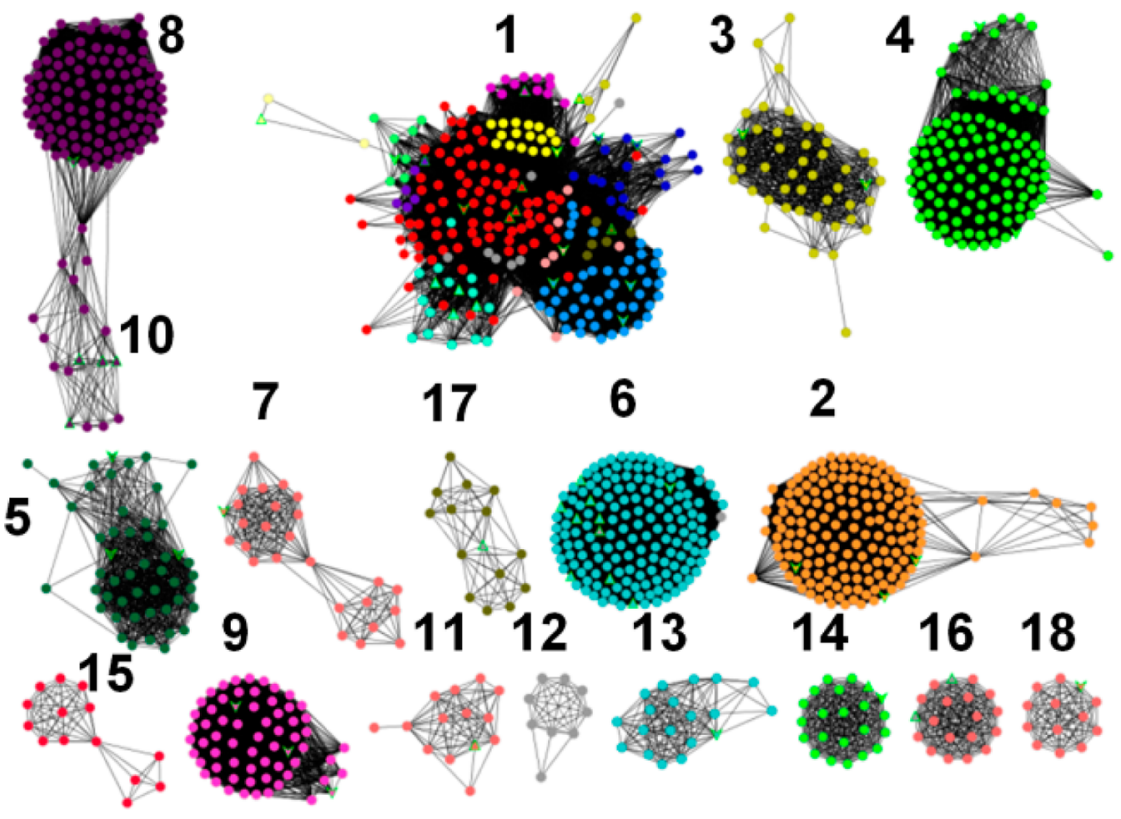

Figure 1. Sequence similarity network for cog0402. Protein sequences for $\operatorname{cog} 0402$ were retrieved from NCBI, subjected to an All-by-All BLAST to determine overall sequence similarity to all of the other proteins within this network. ${ }^{27}$ Each dot (node) represents an enzyme, and each connection between two nodes (an edge) represents those enzyme pairs that are more closely related than the arbitrary $E$-value cutoff $\left(10^{-70}\right)$. Groups are arbitrarily numbered; those groups with experimentally determined substrate profiles are as follows: (1) $S$-adenosylhomocysteine/5'-deoxy-5'methylthioadenosine deaminase (red nodes); (2) guanine deaminase (orange nodes); (4) 8-oxoguanine/isoxanthopterin deaminase (green nodes); (6) cytosine deaminase (light blue nodes); and (8) $\mathrm{N}$-formimino-L-glutamate deiminase (purple nodes).

discovery. ${ }^{15-20}$ For example, this strategy was successfully employed in the prediction of function for $\mathrm{Bc0371}$ from Bacillus cereus as an $\mathrm{N}$-succinyl arginine/lysine racemase by docking dipeptides and $\mathrm{N}$-succinyl amino acids to a homology model of Bc0371 based on the X-ray structure of the closest structural homologue, L-alanine-D/L-glutamate epimerase. ${ }^{21}$

The accuracy of a homology model can be estimated from the target-template sequence identity. When the sequence identity exceeds $30 \%$, a reliable alignment can typically be constructed, and the resulting models may be useful for virtual screening. When the sequence identity decreases much below $30 \%$, the target structure often deviates significantly from that of the template, resulting in large errors in side chain packing, loop conformations, core backbone conformations, alignment, and even fold assignment. Unfortunately, for many proteins from newly sequenced genomes, only distantly related template structures are available. Hence, it is of pressing interest to develop modeling and docking methods that account for backbone variation between homologues, so that homology models based on distant templates can be used for annotation of protein function.

The functionally diverse amidohydrolase superfamily (AHS) of enzymes provides a test case for developing robust computational methods for function identification of uncharacterized enzymes. Enzymes within this superfamily possess a mononuclear or binuclear metal center embedded within a $(\beta /$ $\alpha)_{8}$-barrel structural fold, ${ }^{22}$ and catalyze diverse reactions, including ester and amide hydrolysis, nucleic acid deamination, double bond hydration, carbohydrate isomerization, and decarboxylation. $^{23}$ To date, more than 24000 unique bacterial proteins have been assigned to this superfamily, segregating into 24 clusters of orthologous groups (COGs). ${ }^{24,25}$

One of these COGs, cog0402, contains approximately 1400 distinct proteins. At BLAST ${ }^{26} E$-value of $10^{-70}, \operatorname{cog} 0402$ can be divided into 14 major groups of similar sequences ${ }^{27,28}$ (Figure
1). Although $40 \%$ of the proteins in $\operatorname{cog} 0402$ can be reliably annotated as catalyzing the deamination of an aromatic base or a similar functional group, ${ }^{11,29-34}$ the rest remain uncharacterized. Here, we have attempted to predict the substrate profile for enzymes of unknown function represented by group 14 of $\operatorname{cog} 0402$, containing approximately 140 proteins. In particular, Arad3529 from Agrobacterium radiobacter K84 was cloned, expressed, and purified to homogeneity. Substrates of Arad3529 were predicted by docking high-energy intermediate forms of candidate metabolites to the homology models constructed for Arad3529. High-ranking predictions were acquired and tested as candidate substrates by enzymology. The sequence identity between Arad3529 and its closest structurally characterized homologue, cytosine deaminase from E. coli, is only $26 \%$, making the construction of the homology model and subsequent computational docking highly challenging.

\section{MATERIALS AND METHODS}

General. All chemicals were obtained from Sigma-Aldrich unless otherwise specified. 7,8-Dihydro-L-biopterin was purchased from Santa Cruz Biotechnology. Formylpterin, pterin-7-carboxylate, hydroxymethylpterin, xanthopterin, 7,8-dihydrohydroxymethylpterin, and 7,8dihydroneopterin were purchased from Schirks Laboratories.

Cloning, Expression, and Purification of Arad3529. The gene for Arad3529 was amplified from Agrobacterium radiobacter Strain K84 genomic DNA using 5'-TTAAGAAGGAGATATACCATGTCATACAGTTTCATGTCCCCGCC-3' as the forward primer and $5^{\prime}$ GATTGGAAGTAGAGGTTCTCTGCTGCGCCAATCACGGTGTCCAG-3' as the reverse primer. PCR was performed using KOD Hot Start DNA Polymerase (Novagen). The amplified fragment was cloned into the C-terminal TEV cleavable StrepII-6x-His-tag containing vector, CHS30, by ligation-independent cloning. ${ }^{35}$

The Arad3529-CHS30 vector was used to transform BL21(DE3) E. coli containing the pRIL plasmid (Stratagene), which was used to inoculate a $5 \mathrm{~mL} 2 \mathrm{xYT}$ culture containing $25 \mu \mathrm{g} / \mathrm{mL}$ kanamycin and $34 \mu \mathrm{g} / \mathrm{mL}$ chloramphenicol. The culture was allowed to grow 
overnight at $37^{\circ} \mathrm{C}$ in a shaking incubator. The overnight culture was used to inoculate $1 \mathrm{~L}$ of PASM-5052 autoinduction media ${ }^{36}$ containing $150 \mathrm{mM}$ 2-2-bipyridyl, $1.0 \mathrm{mM} \mathrm{ZnCl}$, and $1.0 \mathrm{mM} \mathrm{MnCl}_{2}$. The culture was placed in a LEX48 airlift fermenter and incubated at $37{ }^{\circ} \mathrm{C}$ for $5 \mathrm{~h}$ and then at $22{ }^{\circ} \mathrm{C}$ overnight. The culture was harvested and pelleted by centrifugation.

Cells were resuspended in lysis buffer (20 mM HEPES pH 7.5, 500 $\mathrm{mM} \mathrm{NaCl}, 20 \mathrm{mM}$ imidazole, and $10 \%$ glycerol) and lysed by sonication. The lysate was clarified by centrifugation at $35000 \mathrm{~g}$ for 30 $\mathrm{min}$. The clarified lysate was loaded onto a $5 \mathrm{~mL}$ Strep-Tactin column (IBA), washed with 5 column volumes of lysis buffer, and then eluted in StrepB buffer (20 mM HEPES pH 7.5, $500 \mathrm{mM} \mathrm{NaCl}, 20 \mathrm{mM}$ imidazole, $10 \%$ glycerol, and $2.5 \mathrm{mM}$ desthiobiotin). The eluent was loaded onto a $1.0 \mathrm{~mL}$ HisTrap FF column (GE Healthcare), washed with 10 column volumes of lysis buffer, and eluted in buffer containing $20 \mathrm{mM}$ HEPES $\mathrm{pH}$ 7.5, $500 \mathrm{mM} \mathrm{NaCl}, 500 \mathrm{mM}$ imidazole, and $10 \%$ glycerol. The purified sample was loaded onto a HiLoad S200 16/60 PR gel filtration column, which was equilibrated with SECB buffer $(20$ $\mathrm{mM}$ HEPES pH7.5, $150 \mathrm{mM} \mathrm{NaCl}, 10 \%$ glycerol, and $5 \mathrm{mM}$ DTT). Peak fractions were collected, analyzed by SDS-PAGE, snap frozen in liquid nitrogen, and stored at $-80{ }^{\circ} \mathrm{C}$. The purified protein was submitted to ICP-MS for metal content analysis and found to contain 0.8 equiv of $\mathrm{Mn}, 0.1$ equiv of $\mathrm{Fe}, 0.1$ equiv of $\mathrm{Ni}$, and 0.1 equiv of $\mathrm{Zn}$.

Homology Modeling of Arad3529. The amino acid sequence of Arad3529 from Agrobacterium radiobacter K84 (gil222086854) was retrieved from the Structure Function Linkage Database (SFLD) ${ }^{37}$ Homology models of Arad3529 were generated in four steps. First, the primary sequence was submitted to the PSI-BLAST server at NCBI to search for suitable template structures. ${ }^{38}$ Cytosine deaminase (CDA) from E. coli (PDB id: 1K70) is the most closely related sequence of known structure. Cytosine deaminase is from group 6 of $\operatorname{cog} 0402$ and shares a 26\% sequence identity to Arad3529 (Figure 1). In this structure, the enzyme is complexed with a mechanism-based inhibitor, 4-(S)-hydroxyl-3,4-dihydropyrimidine. Second, a sequence alignment between Arad3529 and cystosine deaminase was computed by MUSCLE $^{39}$ (Multiple Sequence Comparison by Log-Expectation) (Figure S1). Third, 500 homology models were generated with the standard "automodel" class in MODELLER, ${ }^{40}$ and the model with the best DOPE $^{41}$ score was selected (Model-1) (Figure 2). Finally, two

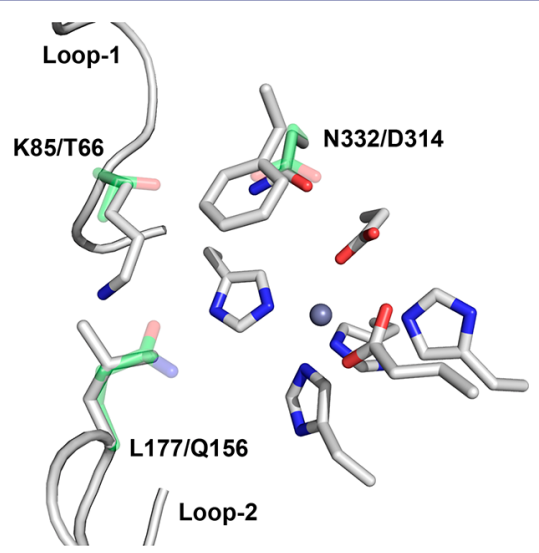

Figure 2. The active site of E. coli $\mathrm{CDA}$ and residues predicted to be important for Arad3529. Residues drawn in white are conserved across all members of $\operatorname{cog} 0402$. Thr-66, Gln-156, and Asp-314 in CDA, displayed in green, differed significantly in the sequence of Arad3529 and may be important for substrate binding. Thr-66 corresponds to Lys-85, Gln-156 corresponds to Leu-177, and Asp-314 corresponds to Asn-332. In the X-ray structure of CDA, Gln-156 forms hydrogen bonds with the bound inhibitor; Asp-314 is found within $4 \AA$ of the CDA inhibitor but only forms steric interactions with it; Thr-66 is more distant from the active site. The conversion of a large lysine in Arad3529 suggests that the Lys- 85 may reach into the active site and play a role in substrate binding. loop regions (residues 83-89, 174-186) in Model-1 were refined with the "loopmodel" class in MODELLER, resulting in Models-2, -3, and -4 (Figure 3). The "loopmodel" class in MODELLER includes several

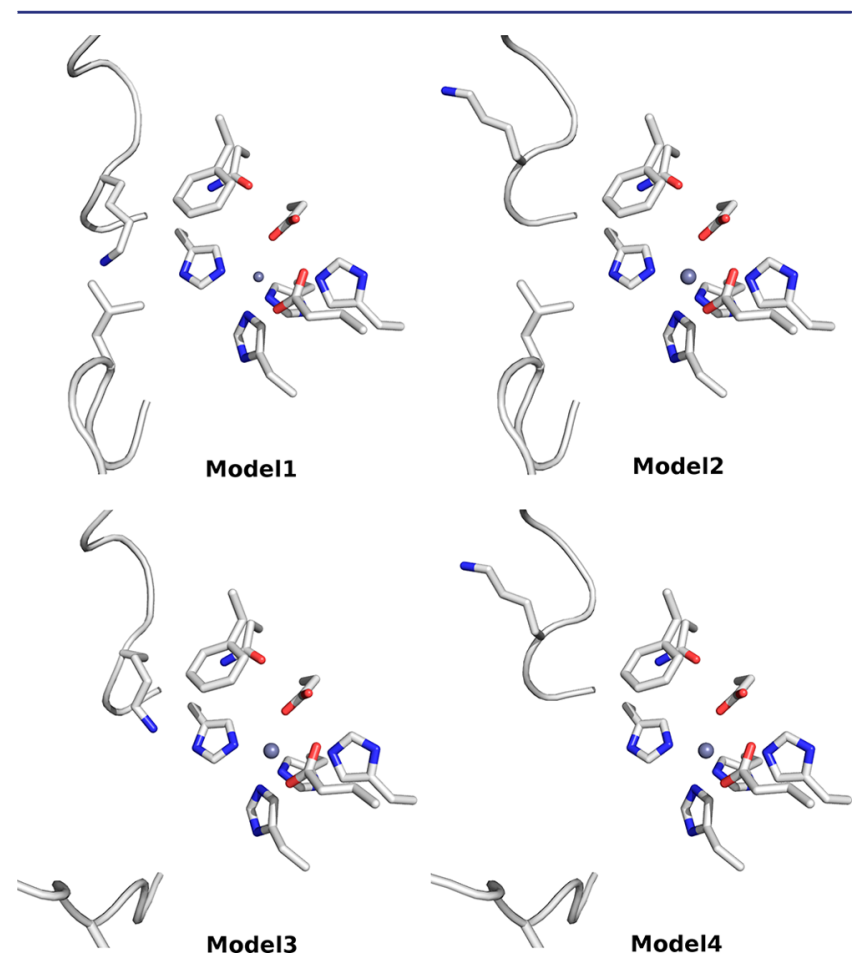

Figure 3. The active site conformations of four representative models of Arad3529 used in virtual screening. Model-1 is ranked the best by DOPE score among 500 homology models generated automatically by MODELLER. Model-2, Model-3, and Model-4 are different from Model-1 in Loop-1, Loop-2, and both Loop-1 and Loop-2, respectively.

loop optimization methods, which all rely on scoring functions and optimization protocols adapted for loop modeling. ${ }^{42}$ Side chains in these two loops were optimized using the "side chain prediction" protocol in PLOP. ${ }^{43}$ The $\mathrm{Fe}^{2+}$ ion was included in all modeling steps. The cocrystallized inhibitor from the template structure was included in the third step for the construction of the initial homology model, but was removed from the modeled active site in the last step.

Docking Screen against Arad3529. A high-energy intermediate (HEI) library ${ }^{44,45}$ that contains 57672 different stereoisomers generated by hydroxide attacking from the $r e$ and $s i$ faces of prochiral molecules and by exploring all possibilities of protonation state of 6440 KEGG (Kyoto Encyclopedia of Genes and Genomes) molecules $^{46}$ was screened against the X-ray structure of the cytosine deaminase template (PDB id: 1K70) as a control, and each of the four homology models of Arad3529, using DOCK 3.6. ${ }^{47}$ The computed poses were subjected to a distance cutoff to ensure that the $\mathrm{O}^{-}$moiety of the HEI portion of the molecule is found within $4 \AA$ of the metal ion in the active site. The top 500 compounds ranked by the docking score (the sum of van der Waals, Poisson-Boltzmann electrostatic, and ligand desolvation penalty terms) were inspected visually to ensure the compatibility of the pose with the amidohydrolase reaction mechanism. The details of the HEI docking library preparation, the molecular docking procedure, and the protocol for analyzing docking results have been previously described., $48-50$

Chemoinformatic Analysis of the Docking Hit-Lists. The top 200 highest ranked compounds for each model were combined into ligand sets and used to calculate self-consistency expectation values $(E$ value) within each set using the Similarity Ensemble Approach (SEA) ${ }^{51,52}$ Briefly, each ligand was broken into molecular fingerprints, here ChemAxon path fingerprints. The similarity between molecules is quantified by the number of bits they have in common divided by the 
Table 1. Virtual Screening of the HEI Database against Four Homology Models of Arad3529 ${ }^{a}$

\begin{tabular}{|c|c|c|c|c|c|}
\hline \multirow[b]{2}{*}{ name } & \multirow[b]{2}{*}{ KEGG ID } & \multicolumn{4}{|c|}{ docking rank } \\
\hline & & Model-1 & Model-2 & Model-3 & Model-4 \\
\hline melamine & C08737 & 10 & & & \\
\hline cyclopropylmelamine & C14147 & 13 & 18 & & \\
\hline diethylatrazine & $\mathrm{C} 06559$ & 28 & 32 & & \\
\hline diisopropylatrazine & C06556 & 29 & 33 & & \\
\hline diisopropylhydroxyatrazine & $\mathrm{C} 06557$ & 31 & & & \\
\hline cytosine & C00380 & 52 & 45 & 185 & 126 \\
\hline 2-amino-4-hydroxy-6-hydroxymethyl-7,8-dihydropteridine & $\mathrm{C} 01300$ & $108(\mathrm{~N} 1)$ & $135(\mathrm{~N} 1)$ & $10(\mathrm{~N} 1)$ & $12(\mathrm{~N} 3)$ \\
\hline thioguanine & C07648 & & 11 & & \\
\hline guanine & $\mathrm{C} 00242$ & & 20 & & \\
\hline \multirow[t]{2}{*}{ pterin } & $\mathrm{C} 00715$ & & $31(\mathrm{~N} 3)$ & $27(\mathrm{~N} 1)$ & $102(\mathrm{~N} 3)$ \\
\hline & & & & & $127(\mathrm{~N} 1)$ \\
\hline phenazopyridine & C07429 & & 50 & & \\
\hline \multirow[t]{2}{*}{ biopterin } & C06313 & & $66(\mathrm{~N} 3)$ & $14(\mathrm{~N} 1)$ & $51(\mathrm{~N} 1)$ \\
\hline & & & & & $71(\mathrm{~N} 3)$ \\
\hline adenine & $\mathrm{C} 00147$ & & 99 & & \\
\hline neopterin & C05926 & & & $15(\mathrm{~N} 1)$ & $143(\mathrm{~N} 1)$ \\
\hline 7-methylguanine & $\mathrm{C} 02242$ & & & & 23 \\
\hline deoxycytidine & $\mathrm{C} 00881$ & & & & 49 \\
\hline cytidine & $\mathrm{C} 00475$ & & & & 75 \\
\hline $5^{\prime}$-deoxy-5'-fluorocytidine & $\mathrm{C} 16635$ & & & & 132 \\
\hline 2-aminoadenosine & C00939 & & & & 206 \\
\hline
\end{tabular}

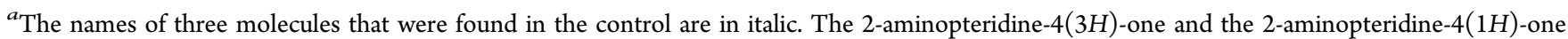
tautomers were attacked by the hydroxide from the re-face and the si-face, and have the $\mathrm{N}-1$ ring nitrogen and $\mathrm{N}$ - 3 ring nitrogen protonated by Glu234, resulting in two intermediates named N1 and N3, respectively.

total number of bits using the Tanimoto coefficient $(T c)$. The sum of all pairwise $T c$ values over a defined cutoff is calculated and compared to what is expected at random between two ligand sets of the same size. The ratio of the calculated sum of $T c$ values over the sum of $T c$ values expected at random is divided by the standard deviation of the random similarity to give a $Z$-score and plotted against an extreme value distribution to yield an $E$-value. The self-similarity between the ligands of the same set provides a metric to how diverse or similar the ligands are based on the $E$-value. The more significant is the $E$-value (lower), the tighter the chemical space is in the ligand set with likely fewer numbers of distinct chemotypes. Each docking model's selfconsistency $E$-value was then compared to the number of true substrates found from each ligand set.

Physical Library Screening. The initial screen of catalytic activity monitored changes in the UV-visible spectrum from 240 to $400 \mathrm{~nm}$ after the addition of $1.0 \mu \mathrm{M}$ Arad3529 to a solution containing 100 $\mu \mathrm{M}$ of the target compound in $20 \mathrm{mM}$ HEPES, $\mathrm{pH} 7.7$ in a 96 -well quartz plate using a Molecular Devices Spectramax 384 Plus spectrophotometer. The compounds that exhibited observable spectral change were subjected to more quantitative kinetic assays. On the basis of the docking ranks and compound availability, 11 compounds prioritized by docking were tested as potential substrates, including melamine, cytosine, thioguanine, guanine, adenine, 7-methylguanine, cytidine, 2'-deoxycytidine, pterin, biopterin, and neopterin. In addition, 104 compounds that were substrates of annotated amidohydrolases were tested as potential substrates (see the Supporting Information). After the pterins were identified as substrates, 11 compounds composed of a pteridine ring were added to the screening.

Determination of Kinetic Constants. Once candidate substrates had been identified in parallel by docking and plate-based screening, quantitative $k_{\text {cat }}$ and $k_{\text {cat }} / K_{\mathrm{m}}$ were determined in $20 \mathrm{mM}$ HEPES buffer at $\mathrm{pH}$ 7.7. For each compound, the change in the extinction coefficient between the substrate and the deaminated product was determined experimentally by subtraction of the absorbance spectrum of the product from the spectrum of the substrate. The wavelengths $(\mathrm{nm})$ and the differential extinction coefficients, $\Delta \varepsilon\left(\mathrm{M}^{-1} \mathrm{~cm}^{-1}\right)$, for each of the compounds utilized in this investigation are as follows: pterin-6- carboxylate $\left(271 \mathrm{~nm}, 6211 \mathrm{M}^{-1} \mathrm{~cm}^{-1}\right)$; pterin $(255$ and $314 \mathrm{~nm}, 7021$, and $\left.1877 \mathrm{M}^{-1} \mathrm{~cm}^{-1}\right)$; biopterin $\left(260 \mathrm{~nm}, 6527 \mathrm{M}^{-1} \mathrm{~cm}^{-1}\right)$; Dneopterin $\left(320 \mathrm{~nm}, 4431 \mathrm{M}^{-1} \mathrm{~cm}^{-1}\right)$; isoxanthopterin $(350 \mathrm{~nm}, 2223$ $\left.\mathrm{M}^{-1} \mathrm{~cm}^{-1}\right)$; sepiapterin $\left(260 \mathrm{~nm}, 3137 \mathrm{M}^{-1} \mathrm{~cm}^{-1}\right)$; folate $(364 \mathrm{~nm}$, $\left.4312 \mathrm{M}^{-1} \mathrm{~cm}^{-1}\right)$; formylpterin $\left(316 \mathrm{~nm}, 2963 \mathrm{M}^{-1} \mathrm{~cm}^{-1}\right)$; pterin-7carboxylate $\left(264 \mathrm{~nm}, 3706 \mathrm{M}^{-1} \mathrm{~cm}^{-1}\right)$; hydroxymethylpterin $(264 \mathrm{~nm}$, $\left.7356 \mathrm{M}^{-1} \mathrm{~cm}^{-1}\right)$; xanthopterin $\left(282 \mathrm{~nm}, 9704 \mathrm{M}^{-1} \mathrm{~cm}^{-1}\right)$; 7,8dihydrohydroxymethylpterin $\left(536 \mathrm{~nm}, 3220 \mathrm{M}^{-1} \mathrm{~cm}^{-1}\right)$; and 7,8dihydroneopterin $\left(282 \mathrm{~nm}, 9424 \mathrm{M}^{-1} \mathrm{~cm}^{-1}\right)$. The values of $k_{\text {cat }}$ and $K_{\mathrm{m}}$ were determined by fitting the parameters in eq 1 to the experimental data, using SigmaPlot 11, where $v$ is the initial velocity, $E_{\mathrm{t}}$ is enzyme concentration, and $A$ is the substrate concentration.

$$
v / E_{\mathrm{t}}=k_{\text {cat }} K_{\mathrm{a}} /\left(A+K_{\mathrm{a}}\right)
$$

Confirmation of Product Formation. $100 \mu \mathrm{M}$ pterin-6carboxylate was incubated with $150 \mathrm{nM}$ Arad3529 in $50 \mathrm{mM}$ $\mathrm{NH} 4 \mathrm{CO} 3 \mathrm{H}$ for $1 \mathrm{~h}$ at $25^{\circ} \mathrm{C}$. The reaction mixture was filtered with a $30000 \mathrm{MW}$ cutoff spin column, and the flow-through was collected. This flow-through and a sample of $100 \mu \mathrm{M}$ pterin-6-carboxylate was submitted to ESI- mass spectrometry at the Texas A\&M Laboratory for biological mass spectrometry. The reaction showed an $\mathrm{m} / z$ change from 206.04 to 207.02 , consistent with the product 2,4-dihydroxypteridine-6-carboxylate from the deamination of pterin-6-carboxylate.

\section{RESULTS}

Sequence Comparison of Arad3529 with Cytosine Deaminase from E. coli. Cytosine deaminase from E. coli is currently the closest structurally characterized protein to Arad3529. The four metal binding residues in cytosine deaminase (H61, H63, H214, and D313) align well with H80, H82, H231, and D331 from Arad3529, along with two additional residues (H246 and E217 aligned with $\mathrm{H} 263$ and E234 in Arad3529, respectively) that function as proton shuttles in cytosine deaminase (Figure S1). Because these active site residues to perform a deamination were all intact in Arad3529, it is very likely also a deaminase. However, in 
Arad3529 there are three notable changes in the amino acid sequence, relative to that found in most other cytosine deaminases from cog0402. In Arad3529, Thr66, Gln156, and Asp314 from CDA are replaced by Lys85, Leu177, and Asn332, respectively (Figure 2). In CDA from E. coli, Thr66 located near the active site does not appear to hydrogen bond with cytosine. Conversely, Gln156 forms a pair of hydrogen bonds to the carbamoyl moiety of cytosine, while Asp314 is located immediately after the invariant Asp313 and within $4 \AA$ of the bound inhibitor in the CDA structure. The substitutions to the active site residues thus suggest that Arad3529 deaminates a substrate different from cytosine.

Molecular Docking. In the initial homology model of Arad3529 (Model-1), which overall adopts the same backbone conformation as the template, changes are located in two unstructured loop regions that help define the active site (Loop-1, residues 83-89; Loop-2, residues 174-186). Therefore, Loop-1 and Loop-2 were remodeled separately, resulting in Model-2 and Model-3 (Figure 3). Finally, the Loop-1 conformation in Model-2 and the Loop-2 conformation in Model-3 were combined in Model-4. Thus, four homology models were obtained that are distinct from each other in their active site conformations.

Because homology models are constructed from template structures, the models often share some structural features with the templates, and thus recognize similar ligands. Here, however, small molecules that are highly ranked by the template structure (PDB id: 1K70) are unlikely to be the true substrate of the remote homologue. Therefore, before targeting the homology models of Arad3529, the template structure $1 \mathrm{~K} 70$ was docked against as a negative control. 57672 high-energy intermediates (HEI) were docked into the template structure. As expected, cytosine, the natural substrate of the template, was ranked highly (10th out of 57672 ), as were several other nucleosides (Table S1). These molecules were deprioritized for testing against Arad3529; we instead looked for molecules with high differential ranks between the models and the template docking screens.

The same HEI database was docked to each of the four homology models of Arad3529. From each model, putative substrates were selected from the top 500 scored molecules (Table 1). The compound sets selected using different homology models are overlapping but distinct. For Model-1, the docking hits were dominated by relatively small compounds with a 1,3,5-triazine skeleton. This pattern is also observed in the docking hits from Model-2. However, compounds composed of the purine or pteridine ring were also favored by Model-2. For Model-3, the 1,3,5-triazine skeleton completely disappeared, and the docking hits were dominated by compounds composed of the pteridine ring. Model-4 shared the pteridine pattern with Model-3, but also favored nucleosides having a ribose group.

Chemoinformatic Analysis of the Docking Hit-Lists. On the basis of docking energies alone, it is difficult to prioritize one of these overall hit-lists over another, although the geometry of the interactions does provide guidance (below). One can, however, pose extra-thermodynamic criteria to judge among them. One such is to expect that in a well-behaved docking hit-list, the highly ranked molecules will resemble one another, and thus the hit-list will resemble itself by chemical similarity. We therefore compared the top-ranked 200 molecules for each of the four models of Arad3529 against themselves using the Similarity Ensemble Approach (SEA). ${ }^{51,52}$
This was quantified by an expectation value (E-value) of the likelihood the self-similarity of each hit-list would occur at random. Of the four models, Model-3 and Model-4 had more self-consistent hit-lists (SEA E-value $1.5 \times 10^{-100}$ and $4.9 \times$ $10^{-98}$, respectively) as compared to Model-1 and Model-2 (SEA E-value $2.7 \times 10^{-89}$ and $1.2 \times 10^{-85}$, respectively). Comfortingly, the structures of what turned out to be the true pterin substrates of the enzyme were docked in catalytically competent geometries in Model-3 and Model-4. Naturally, this criterion has nothing to recommend it other than consistency, but it may merit further study as a metric to select multiple possible hit-lists for targets adopting different structures.

Substrate Profile for Arad3529. On the basis of commercial availability, eight compounds predicted to be potential substrates for Arad3529 were tested as enzyme substrates, as were three "negative control" nucleosides that also ranked well against the CDA template (Table 1). Arad3529 was found to deaminate three of the eight: pterin, biopterin, and neopterin with $k_{\mathrm{cat}} / K_{\mathrm{m}}$ values of greater than $10^{6} \mathrm{M}^{-1} \mathrm{~s}^{-1}$. We docked the ground states of three substrates, pterin, biopterin, and neopterin, to Model-3. The docking scores of these ground states $(-19.29,-27.30$, and -23.08 , respectively) were substantially higher (worse) than the corresponding HEI states $(-53.83,-58.45$, and -58.26 , respectively), consistent with the catalytic mechanism. The pterin deaminase (PDA) reaction is illustrated in Scheme 1 . To investigate the

Scheme 1

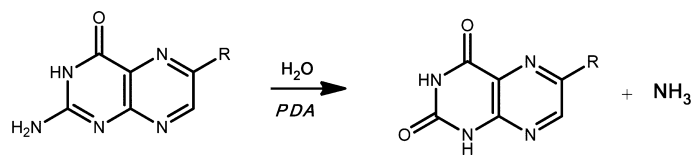

mechanism further, the enzyme was screened with other substituted pterins and was found to deaminate several pterins (Scheme S1). Various substitutions on C6 of the pteridine ring are allowed (Table 2), and with the exception of sepiapterin, the fully aromatic pteridine ring is preferred. 7,8-Dihydroneopterin and 7,8-dihydrobiopterin were deaminated very slowly, and the deamination of dihydrofolate or tetrahydrofolate could not be measured $\left(<0.0008 \mathrm{~s}^{-1}\right)$. 2,4-Diamino-6hydroxymethylpteridine was not deaminated.

Variations on Binding Orientation of Pterin. In all of the structurally characterized members of the amidohydrolase superfamily, the re-face of the carbonyl group of amide and ester substrates is presented to the attacking hydroxide; the same orientation was observed for the amidine or guanidine moieties of substrates in the deamination reactions. In docking screens against Arad3529, we observed two chiral intermediates of pterin (named as N1 and N3) that were generated by hydroxide $r e$ - and si-face attacking two pterin tautomers 2aminopteridin-4 $(3 \mathrm{H})$-one and 2 -aminopteridin-4( $1 \mathrm{H})$-one, and by Glu234 protonating the $\mathrm{N}-1$ and $\mathrm{N}-3$ nitrogen in the pteridine ring, respectively (Scheme 2). In particular, N3 was selected by Model-2, N1 was selected by Model-3, and both intermediates were selected by Model-4 (Table 1). In Model-2, the docking pose of N3 had a stranded C-4 carbonyl group, which in N1 forms hydrogen bonds with Asn332 in Model-3 (Figure 4). This C-4 carbonyl group and the N-5 ring nitrogen in $\mathrm{N} 1$ also hydrogen bonded with Lys85 in Model-3. Furthermore, it is known that the 2-aminopteridin-4(1H)-one tautomer is less stable than the 2-aminopteridin-4(3H)-one 
Table 2. Catalytic Constants for Substrates of Arad3529

\begin{tabular}{|c|c|c|c|c|}
\hline substrate & $k_{\text {cat }} / K_{\mathrm{m}}\left(\mathrm{M}^{-1} \mathrm{~s}^{-1}\right)$ & $k_{\text {cat }}\left(\mathrm{s}^{-1}\right)$ & $K_{\mathrm{m}}(\mu \mathrm{M})$ & docking rank $^{a}$ \\
\hline formylpterin & $5.2(0.5) \times 10^{6}$ & $64 \pm 12$ & $12 \pm 2$ & \\
\hline pterin-6-carboxylate & $4.0(0.2) \times 10^{6}$ & $110 \pm 3$ & $27 \pm 2$ & \\
\hline pterin-7-carboxylate & $3.7(0.2) \times 10^{6}$ & $48 \pm 2$ & $13 \pm 1$ & \\
\hline pterin & $3.3(0.3) \times 10^{6}$ & $131 \pm 4$ & $39 \pm 4$ & 27 \\
\hline hydroxymethylpterin & $1.2(0.1) \times 10^{6}$ & $28 \pm 1$ & $23 \pm 3$ & \\
\hline biopterin & $1.0(0.1) \times 10^{6}$ & $46 \pm 4$ & $47 \pm 9$ & 14 \\
\hline $\mathrm{D}-(+)$-neopterin & $3.1(0.1) \times 10^{5}$ & $19 \pm 1$ & $61 \pm 7$ & 15 \\
\hline isoxanthopterin & $2.8(0.2) \times 10^{5}$ & $1.6 \pm 0.1$ & $5.7 \pm 0.5$ & \\
\hline sepiapterin & $1.3(0.4) \times 10^{5}$ & $2.9 \pm 0.3$ & $22 \pm 9$ & \\
\hline folate & $1.3(0.2) \times 10^{5}$ & $6.4 \pm 0.5$ & $50 \pm 10$ & \\
\hline xanthopterin & $1.2(0.1) \times 10^{5}$ & $0.46 \pm 0.01$ & $40 \pm 1$ & \\
\hline 7,8-dihydro-hydroxymethylpterin & $3.3(0.4) \times 10^{4}$ & $1.2 \pm 0.1$ & $37 \pm 7$ & \\
\hline 7,8-dihydroneopterin & $2.6(0.3) \times 10^{2}$ & $0.036 \pm 0.008$ & $200 \pm 100$ & \\
\hline 7,8-dihydrobiopterin & $9.3(0.5) \times 10^{2}$ & $0.090 \pm 0.007$ & $93 \pm 13$ & \\
\hline
\end{tabular}

${ }^{a}$ In docking against Model-3 (Table 1), compounds were ranked out of the 57672 high-energy intermediates in the virtual library, which only contains pterin, biopterin, and neopterin among the pterin substrates.

Scheme 2

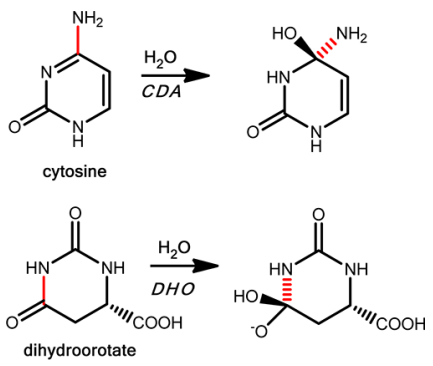<smiles>Nc1nc2nccnc2c(=O)[nH]1</smiles><smiles>N[C@@]1(O)NC(=O)c2nccnc2N1</smiles>

2-aminopteridin-4( $3 \mathrm{H})$-one<smiles>Nc1nc(=O)c2nccnc2[nH]1</smiles>

2-aminopteridin-4( $1 \mathrm{H})$-one<smiles>N[C@@]1(O)NC(=O)c2nccnc2N1</smiles>

tautomer (in ab initio and free energy perturbation calculations, the ratio is $1: 6) .{ }^{53}$ It seems clear that the $\mathrm{N} 1$ orientation is the true binding motif, and that an important feature for recognition of a pterin deaminase is the DN dyad (D331, $\mathrm{N} 332$ ) found on $\beta$-strand 8 and the Lys 85 found on $\beta$-strand 1 .

\section{DISCUSSION}

Three techniques were crucial for what turned out to be the correct prediction of pterin deaminase activity for Arad3529. First, to leverage a distantly related template, detailed modeling of two active-site loops was required to find models that were at once structurally sensible and catalytically competent, and that could discriminate new substrates from these from the template. Second, the selectivity of models was enhanced by negative control docking screens against the template structure; we sought substrates ranked highly by the models and poorly by the template. Finally, a close cycle of bioinformatics, biophysical modeling, and enzymology made this approach pragmatic. Identifying substrates for Arad3529 allows the

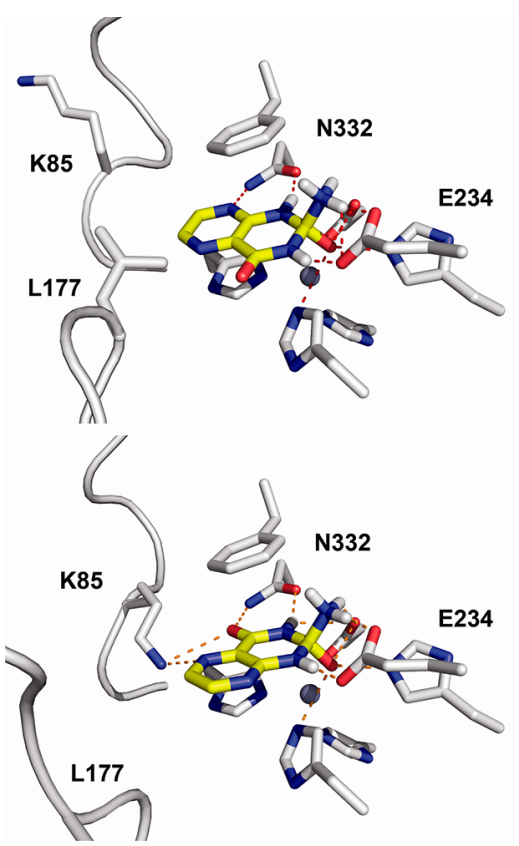

Figure 4. (Top) The binding pose of pterin intermediate N3 (yellow stick) formed by the activated hydroxide attacking the C-2 atom on the $s i$-face of the aromatic ring of the tautomer 2-aminopteridin4(1H)-one, in the active site of Model-2 of Arad3529 (white stick). (Bottom) The binding pose of pterin intermediate N1 (yellow stick) formed by the activated hydroxide attacking the $\mathrm{C}-2$ atom on the reface of the aromatic ring of the tautomer 2-aminopteridin-4(3H)-one, in the active site of Model-3 of Arad3529 (white stick). Polar interactions between the docked pterin and the modeled active site are shown by red dashed lines.

functional annotation of about 140 previously uncharacterized amidohydrolase enzymes in group 14 of cog0402. These proteins may be used by bacteria to salvage oxidized pterins, and form the pterin degradation pathway with neighboring genes.

Enzyme Specificity Recapitulated by Loop Modeling. The substrate specificity of enzymes comes from the corresponding compositions of binding-site residues. Remarkable changes in the binding-site residues between two enzyme sequences lead to differences in their binding-site structures. However, a homology model tends to inherit the backbone 
conformations of aligned regions from the template. Therefore, a homology model of the target enzyme often contains errors in the packing of side chains, and backbone conformations of structurally undefined regions (loops), especially when computed on the basis of a remote template. Accurate substrate prediction by docking against homology models of unknown enzymes requires precise positioning of the binding site residues that interact with the new substrates. In this study, we attempted to capture binding-site features in Arad3529 by remodeling two binding-site loops in the initial homology model. Because no target ligand is known, four models that are structurally diverse were selected and docked against independently, followed by combining the screening results. True substrates of Arad3529, pterins, were not captured by the initial homology model, but were captured by the models with refined loops, illustrating the necessity of the directed refinement of binding-site loops that contain residues different from the template.

Docking against the Template as a Negative Control. When a homology model of the target enzyme is computed on the basis of a distantly related template structure, on one side, the target enzyme very likely has different substrates from those of the template; on the other side, however, the homology model of the target even after refinement could still share some structural features with the template and thus prioritize similar compounds as the template structure in docking screens. Here, we first docked to the template as a negative control, and compared these hits to those against the homology models. Compounds that were ranked highly by the template and by the homology models were discounted, and indeed none of them was confirmed as substrates of Arad3529. This suggests that, for enzyme substrate prediction by homology model-based docking, it may be useful to account for compounds that are recognized by the template as negative control, especially when there is only a remote relationship between the target and the template sequence.

Additional Enzymes in cog0402 That Catalyze Pterin Deamination. Figure 1 depicts 22 sequences in group 14. To identify a more comprehensive list of proteins in the current databases that share the same substrate profile as Arad3529, a BLAST search was conducted with the sequence of Arad3529. Of the top 200 hits, 165 proteins contained the characteristic $\beta$ strand 8 "DN" dyad, and a total of 139 of these would populate group 14 if included in the network diagram. The remaining 26 sequences cluster with the currently uncharacterized group 13 . Group 13 is closely related to cytosine deaminase and also possesses a mixture of enzymes that have either a "DN" or "NN" dyad. While the enzymatic function of group 13 is unknown, all members of group 14 are predicted to deaminate pterins (Table S2). Of course, this remains just a prediction, because even proteins with high sequence identity sometimes have different activities. ${ }^{54}$

Biological Relevance of Substrates, Genomic Context in Agrobacterium radiobacter. Pterin rings form the backbone of the coenzymes folate and tetrahydrobiopterin. The 7,8-dihydro forms of hydroxymethylpterin and neopterin are intermediates in folate synthesis, whereas sepiapterin is an intermediate in the synthesis of biopterin. During the biosynthesis of these cofactors, the pterin substructure remains reduced in the 7,8-dihydro form. Fully oxidized pterins, formed by oxidation of 7,8 -dihydropterins, ${ }^{55}$ are not known to be salvaged by reduction. ${ }^{56}$ Isoxanthopterin is formed by oxygenation of pterin by xanthine dehydrogenase, ${ }^{57}$ while pterin-6- carboxylate, formylpterin, and pterin can be formed by photolysis of folate. $^{58,59}$ It seems possible that Arad3529 and its orthologues are used by the bacteria to degrade oxidized pterins.

The gene for Arad3529 is situated in an apparent operon along with the genes $\mathrm{TauC}, \mathrm{GlcD}$, and $\mathrm{MdaB}$, which are flanked by TauA, TauB, LysR, and a protein from $\operatorname{cog} 1402$ (Figure S2). TauA, TauB, and TauC resemble ATP-cassette binding transporters, and LysR is a transcription factor. $\mathrm{MdaB}, \mathrm{GlcD}$, and $\operatorname{cog} 1402$ are uncharacterized proteins resembling quinone oxidase, glycolate oxidase $\mathrm{D}$, and creatininase, respectively. $\mathrm{MdaB}$ is predicted to contain an $\mathrm{NAD}(\mathrm{P}) \mathrm{H}$ binding domain, GlcD is predicted to be a flavin-dependent redox enzyme, and the $\operatorname{cog} 1402$ protein will likely catalyze the hydrolysis of an amide bond. These enzymes may form a degradative pathway for catabolizing pterin rings. To our knowledge, the only known instance for the degradation of a pterin ring is by the soil bacteria Alcaligenes faecalis. ${ }^{60}$ In this bacterium, isoxanthopterin is deaminated to form 7-oxylumazine, which is further oxidized to 6,7-dioxylumazine (tetraoxypteridine). An isomerase then cleaves the $\mathrm{C} 6 / \mathrm{C} 7$ bond, and reattaches, forming xanthine-8-carboxylate. ${ }^{61}$ Xanthine-8-carboxylate is then decarboxylated, forming xanthine. ${ }^{62}$ Unfortunately, the recently sequenced genome of $A$. faecalis does not possess an Arad3529 homologue or any of its operon-related genes, and the protein that deaminates isoxanthopterin remains to be discovered.

Certain caveats merit mentioning. Here, homology models of Arad3529 were predicted from a cytosine deaminase structure in complex with a mechanism-based inhibitor. This likely gave us an advantage over modeling from a ligand-free template. When such an apo- template is the only choice, enhanced sampling may be needed. ${ }^{19}$ Inevitably, this will lead to more models. To judge among these possible structures, and the docked molecules to which they lead, the criterion of a selfsimilar hit-list may be useful, as we found it to be. Admittedly, this metric remains largely untested, and its only theoretical virtue is that it quantifies an expectation that a well-behaved docking calculation should find putative ligands that resemble one another. From a docking standpoint, we have continued to use high-energy intermediate forms of substrates, rather than their ground states. This seems well-justified for amidohydrolases, where we have a good understanding of the structures that such intermediates adopt, and confidence that the chemical step is rate determining for the reactions the enzymes catalyze. For other families of enzymes, our ability to anticipate highenergy structures, and confidence that the chemical step is rate limiting, may be more limited. Finally, docking screens, in our hands, retain a human element as a final arbiter of which among the tens-to-hundreds of high-ranking metabolites to actually test experimentally. We have argued that human inspection integrates aspects of docking that are captured by the experience of the trained modeler, enzymologist, or medicinal chemist that are difficult to fully capture algorithmically. ${ }^{63}$

\section{- CONCLUSION}

Members of the amidohydrolase superfamily have been found in every sequenced genome; the functions of most of these proteins remain unknown. Here, we focused on one amidohydrolase, Arad3529 from Agrobacterium radiobacter K84, which represents proteins from group 14 in cog0402. Proteins in group 14 were uncharacterized, with sequence similarity and knowledge of the mechanistically related 
chemistries within the superfamily wrongly suggesting roles as chlorohydrolases or cytosine deaminases. To determine the function of Arad3529, we used a multidisciplinary approach that integrated homology modeling, molecular docking screens of a metabolite library, and physical library screening by kinetic assays. This led to what we believe are the true substrates of Arad3529, a set of modified pterins. On the basis of the conservation of characteristic residues that interact with substrates in the docked structure, about 140 other previously unannotated amidohydrolases from different species may now be assigned as pterin deaminases. This approach may be useful in the discovery of in vitro enzymatic and in vivo metabolical functions of unknown enzymes discovered in genome projects, especially for those targets with marginal sequence identities to template structures of known function.

\section{ASSOCIATED CONTENT}

\section{S Supporting Information}

Compounds tested in physical library screening. This material is available free of charge via the Internet at http://pubs.acs.org.

\section{AUTHOR INFORMATION}

\section{Corresponding Author}

raushel@tamu.edu; shoichet@cgl.ucsf.edu; sali@salilab.org

\section{Author Contributions}

$\nabla_{\text {These authors contributed equally. }}$

\section{Notes}

The authors declare no competing financial interest.

\section{ACKNOWLEDGMENTS}

This work was supported by NIH grants (GM071790 and U54 GM093342; R01 GM054762 to A.S.) and the Robert A. Welch Foundation (A-840 to F.M.R.). We thank Dr. Patricia Babbitt and Dr. Sunil Ojha for discussion about amidohydrolse superfamily and sequence analysis. We thank Dr. Peter Kolb and Dr. Magdalena Korczynska for discussion about the highenergy intermediate (HEI) docking database.

\section{REFERENCES}

(1) Whisstock, J. C.; Lesk, A. M. Q. Rev. Biophys. 2003, 36, 307.

(2) Gerlt, J. A.; Babbitt, P. C. Genome Biol. 2000, 1.

(3) Brenner, S. E. Trends Genet. 1999, 15, 132.

(4) Devos, D.; Valencia, A. Trends Genet. 2001, 17, 429.

(5) Gerlt, J. A.; Allen, K. N.; Almo, S. C.; Armstrong, R. N.; Babbitt, P. C.; Cronan, J. E.; Dunaway-Mariano, D.; Imker, H. J.; Jacobson, M. P.; Minor, W.; Poulter, C. D.; Raushel, F. M.; Sali, A.; Shoichet, B. K.; Sweedler, J. V. Biochemistry 2011, 50, 9950.

(6) Schnoes, A. M.; Brown, S. D.; Dodevski, I.; Babbitt, P. C. PLoS Comput. Biol. 2009, 5, e1000605.

(7) Glasner, M. E.; Fayazmanesh, N.; Chiang, R. A.; Sakai, A.; Jacobson, M. P.; Gerlt, J. A.; Babbitt, P. C. J. Mol. Biol. 2006, 360, 228.

(8) Favia, A. D.; Nobeli, I.; Glaser, F.; Thornton, J. M. J. Mol. Biol. 2008, 375, 855 .

(9) Hermann, J. C.; Marti-Arbona, R.; Fedorov, A. A.; Fedorov, E.; Almo, S. C.; Shoichet, B. K.; Raushel, F. M. Nature 2007, 448, 775.

(10) Cummings, J. A.; Nguyen, T. T.; Fedorov, A. A.; Kolb, P.; Xu, C. F.; Fedorov, E. V.; Shoichet, B. K.; Barondeau, D. P.; Almo, S. C.; Raushel, F. M. Biochemistry 2010, 49, 611.

(11) Hall, R. S.; Fedorov, A. A.; Marti-Arbona, R.; Fedorov, E. V.; Kolb, P.; Sauder, J. M.; Burley, S. K.; Shoichet, B. K.; Almo, S. C.; Raushel, F. M. J. Am. Chem. Soc. 2010, 132, 1762.

(12) Xiang, D. F.; Kolb, P.; Fedorov, A. A.; Xu, C. F.; Fedorov, E. V.; Narindoshivili, T.; Williams, H. J.; Shoichet, B. K.; Almo, S. C.; Raushel, F. M. Biochemistry 2012, 51, 1762.
(13) Marti-Renom, M. A.; Stuart, A. C.; Fiser, A.; Sanchez, R.; Melo, F.; Sali, A. Annu. Rev. Biophys. Biomol. 2000, 29, 291.

(14) Pieper, U.; Eswar, N.; Webb, B. M.; Eramian, D.; Kelly, L.; Barkan, D. T.; Carter, H.; Mankoo, P.; Karchin, R.; Marti-Renom, M. A.; Davis, F. P.; Sali, A. Nucleic Acids Res. 2009, 37, D347.

(15) Evers, A.; Klebe, G. J. Med. Chem. 2004, 47, 5381.

(16) Kairys, V.; Fernandes, M. X.; Gilson, M. K. J. Chem. Inf. Model. 2006, 46, 365.

(17) Katritch, V.; Byrd, C. M.; Tseitin, V.; Dai, D. C.; Raush, E.; Totrov, M.; Abagyan, R.; Jordan, R.; Hruby, D. E. J. Comput.-Aided Mol. Des. 2007, 21, 549.

(18) Fan, H.; Irwin, J. J.; Webb, B. M.; Klebe, G.; Shoichet, B. K.; Sali, A. J. Chem. Inf. Model. 2009, 49, 2512.

(19) Carlsson, J.; Coleman, R. G.; Setola, V.; Irwin, J. J.; Fan, H.; Schlessinger, A.; Sali, A.; Roth, B. L.; Shoichet, B. K. Nat. Chem. Biol. 2011, 7, 769.

(20) Schlessinger, A.; Geier, E.; Fan, H.; Irwin, J. J.; Shoichet, B. K.; Giacomini, K. M.; Sali, A. Proc. Natl. Acad. Sci. U.S.A. 2011, 108, 15810 .

(21) Song, L.; Kalyanaraman, C.; Fedorov, A. A.; Fedorov, E. V.; Glasner, M. E.; Brown, S.; Imker, H. J.; Babbitt, P. C.; Almo, S. C.; Jacobson, M. P.; Gerlt, J. A. Nat. Chem. Biol. 2007, 3, 486.

(22) Holm, L.; Sander, C. Proteins: Struct., Funct., Bioinf. 1997, 28, 72.

(23) Seibert, C. M.; Raushel, F. M. Biochemistry 2005, 44, 6383.

(24) Tatusov, R. L.; Koonin, E. V.; Lipman, D. J. Science 1997, 278, 631.

(25) Pieper, U.; Chiang, R.; Seffernick, J. J.; Brown, S. D.; Glasner, M. E.; Kelly, L.; Eswar, N.; Sauder, J. M.; Bonanno, J. B.; Swaminathan, S.; Burley, S. K.; Zheng, X.; Chance, M. R.; Almo, S. C.; Gerlt, J. A.; Raushel, F. M.; Jacobson, M. P.; Babbitt, P. C.; Sali, A. J. Struct. Funct. Genomics 2009, 10, 107.

(26) Altschul, S. F.; Gish, W.; Miller, W.; Myers, E. W.; Lipman, D. J. J. Mol. Biol. 1990, 215, 403.

(27) Atkinson, H. J.; Morris, J. H.; Ferrin, T. E.; Babbitt, P. C. PLoS One 2009, 4, e4345.

(28) Cline, M. S.; Smoot, M.; Cerami, E.; Kuchinsky, A.; Landys, N.; Workman, C.; Christmas, R; Avila-Campilo, I.; Creech, M.; Gross, B.; Hanspers, K.; Isserlin, R.; Kelley, R.; Killcoyne, S.; Lotia, S.; Maere, S.; Morris, J.; Ono, K.; Pavlovic, V.; Pico, A. R.; Vailaya, A.; Wang, P.-L.; Adler, A.; Conklin, B. R.; Hood, L.; Kuiper, M.; Sander, C.; Schmulevich, I.; Schwikowski, B.; Warner, G. J.; Ideker, T.; Bader, G. D. Nat. Protoc. 2007, 2, 2366.

(29) Maynes, J. T.; Yuan, R. G.; Snyder, F. F. J. Bacteriol. 2000, 182, 4658.

(30) Yuan, G.; Bin, J. C.; McKay, D. J.; Snyder, F. F. J. Biol. Chem. 1999, 274, 8175

(31) Ireton, G. C.; McDermott, G.; Black, M. E.; Stoddard, B. L. J. Mol. Biol. 2002, 315, 687.

(32) Danielsen, S.; Kilstrup, M.; Barilla, K.; Jochimsen, B.; Neuhard, J. Mol. Microbiol. 1992, 6, 1335.

(33) Hall, R. S.; Agarwal, R.; Hitchcock, D.; Sauder, J. M.; Burley, S. K.; Swaminathan, S.; Raushel, F. M. Biochemistry 2010, 49, 4374.

(34) Marti-Arbona, R.; Xu, C. F.; Steele, S.; Weeks, A.; Kuty, G. F.; Seibert, C. M.; Raushel, F. M. Biochemistry 2006, 45, 1997.

(35) Aslanidis, C.; Dejong, P. J. Nucleic Acids Res. 1990, 18, 6069.

(36) Studier, F. W. Protein Expression Purif. 2005, 41, 207.

(37) Pegg, S. C. H.; Brown, S. D.; Ojha, S.; Seffernick, J.; Meng, E. C.; Morris, J. H.; Chang, P. J.; Huang, C. C.; Ferrin, T. E.; Babbitt, P. C. Biochemistry 2006, 45, 2545.

(38) Altschul, S. F.; Madden, T. L.; Schaffer, A. A.; Zhang, J. H.; Zhang, Z.; Miller, W.; Lipman, D. J. Nucleic Acids Res. 1997, 25, 3389.

(39) Edgar, R. C. Nucleic Acids Res. 2004, 32, 1792.

(40) Sali, A.; Blundell, T. L. J. Mol. Biol. 1993, 234, 779.

(41) Shen, M. Y.; Sali, A. Protein Sci. 2006, 15, 2507.

(42) Fiser, A.; Do, R. K. G.; Sali, A. Protein Sci. 2000, 9, 1753.

(43) Sherman, W.; Day, T.; Jacobson, M. P.; Friesner, R. A.; Farid, R. J. Med. Chem. 2006, 49, 534.

(44) Hermann, J. C.; Ghanem, E.; Li, Y. C.; Raushel, F. M.; Irwin, J. J.; Shoichet, B. K. J. Am. Chem. Soc. 2006, 128, 15882. 
(45) Xiang, D. F.; Kolb, P.; Fedorov, A. A.; Meier, M. M.; Fedorov, L. V.; Nguyen, T. T.; Sterner, R.; Almo, S. C.; Shoichet, B. K.; Raushel, F. M. Biochemistry 2009, 48, 2237.

(46) Kanehisa, M.; Goto, S.; Hattori, M.; Aoki-Kinoshita, K. F.; Itoh, M.; Kawashima, S.; Katayama, T.; Araki, M.; Hirakawa, M. Nucleic Acids Res. 2006, 34, D354.

(47) Mysinger, M. M.; Shoichet, B. K. J. Chem. Inf. Model. 2010, 50, 1561.

(48) Goble, A. M.; Fan, H.; Sali, A.; Raushel, F. M. ACS Chem. Biol. 2011, 6, 1036.

(49) Kamat, S. S.; Bagaria, A.; Kumaran, D.; Holmes-Hampton, G. P.; Fan, H.; Sali, A.; Sauder, J. M.; Burley, S. K.; Lindahl, P. A.; Swaminathan, S.; Raushel, F. M. Biochemistry 2011, 50, 1917.

(50) Kamat, S. S.; Fan, H.; Sauder, J. M.; Burley, S. K.; Shoichet, B. K.; Sali, A.; Raushel, F. M. J. Am. Chem. Soc. 2011, 133, 2080.

(51) Keiser, M. J.; Roth, B. L.; Armbruster, B. N.; Ernsberger, P.; Irwin, J. J.; Shoichet, B. K. Nat. Biotechnol. 2007, 25, 197.

(52) Hert, J.; Keiser, M. J.; Irwin, J. J.; Oprea, T. I.; Shoichet, B. K. J. Chem. Inf. Model. 2008, 48, 755.

(53) Schwalbe, C. H.; Lowis, D. R.; Richards, W. G. J. Chem. Soc. Chem. Commun. 1993, 1199.

(54) Seffernick, J. L.; de Souza, M. L.; Sadowsky, M. J.; Wackett, L. P. J. Bacteriol. 2001, 183, 2405.

(55) Pfleiderer, W. Folates Pterins 1984, 2, 43.

(56) Noiriel, A.; Naponelli, V.; Gregory, J. F.; Hanson, A. D. Plant Physiol. 2007, 143, 1101.

(57) Forrest, H. S.; Glassman, E.; Mitchell, H. K. Science 1956, 124, 725 .

(58) Lowry, O. H.; Bessey, O. A.; Crawford, E. J. J. Biol. Chem. 1949, $180,389$.

(59) Off, M. K.; Steindal, A. E.; Porojnicu, A. C.; Juzeniene, A.; Vorobey, A.; Johnsson, A.; Moan, J. J. Photochem. Photobiol., B 2005, $80,47$.

(60) McNutt, W. S., Jr. J. Biol. Chem. 1968, 238, 1116.

(61) McNutt, W. S.; Damle, S. P. J. Biol. Chem. 1964, 239, 4272.

(62) Dairman, W. M.; McNutt, W. S. J. Biol. Chem. 1964, 239, 3407.

(63) Mysinger, M. M.; Weiss, D. R.; Ziarek, J. J.; Gravel, S.; Doak, A. K.; Karpiak, J.; Heveker, N.; Shoichet, B. K.; Volkman, B. F. Proc. Natl. Acad. Sci. U.S.A. 2012, 109, 5517. 